\section{G167(P) THE MULTI-DISCIPLINARY PAEDIATRIC TRACHEOSTOMY CLINIC}

doi:10.1136/archdischild-2013-304107.179

'MMC Yaneza, 2P Davies, ${ }^{3} \mathrm{~S}$ Harrison, 'WA Clement, 'H Kubba. 'Department of Otolaryngology, Royal Hospital for Sick Children, Yorkhill, Glasgow, UK; ${ }^{2}$ Department of Paediatric Respiratory Medicine, Royal Hospital for Sick Children, Yorkhill, Glasgow, UK; ${ }^{3}$ Royal Hospital for Sick Children, Yorkhill, Glasgow, UK

Aims To present our experience of managing children with a tracheostomy in a Multi-Disciplinary Team clinic consisting of an ENT Consultant, Paediatric Respiratory Consultant, a Nurse Specialist and Speech and Language Therapist.

Methods Retrospective case note review of all children seen in the Multi-Disciplinary Team Tracheostomy clinic between February 2009 and May 2011. Data analysed to determine the current underlying indication for tracheostomy and the number of tracheostomy related issues managed per clinical episode.

Results The MDT Tracheostomy clinic began in February 2009 and is held once a month. 44 different patients have been seen in 81 outpatient episodes between February 2009 and May 2011. In our series the three most common indications for tracheostomy were; underlying neurodevelopmental problems $(61.3 \%, n=27)$, most commonly cerebral palsy $(43.2 \%, n=9)$, lower airway problems $(59 \%, n=26)$ and upper airway obstruction (including subglottic stenosis, $n=7)(59 \%, n=26) .15$ patients were on ventilators with a further 6 patients who had had previously been on long term ventilation. The median number of tracheostomy related issues dealt with each appointment was 1 (range $0-4$ ). Secretions were the most common problem (51.7\% of visits). $40 \%(n=6)$ patients on a ventilator had ventilator issues reviewed. 14 patients had a speaking valve already and 10 were considered for a trial of a speaking valve.

Conclusions Children with a tracheostomy are a diverse group of patients. The most common indications for paediatric tracheostomy have changed in the last 50 years from infective causes to airway obstruction and anomalies, long-term ventilation requirement and underlying neuromuscular or respiratory problems. Our approach to managing this diverse group of patients involves a Multi-Disciplinary Team consisting of a Paediatric Otolaryngology Consultant, a Paediatric Respiratory Physician and a Tracheostomy Nurse Specialist or Speech and Language Therapist (SALT). The unified approach also empowers the carers and patient as a home management plan, long-term plan and goals are generated at the end of each appointment.

\section{G168(P) A REVIEW OF THE EFFECTIVENESS OF HEATED, HUMIDIFIED HIGH FLOW NASAL CANNULA THERAPY (HHHFNC) FOR PATIENTS WITH BRONCHIOLITIS}

doi:10.1136/archdischild-2013-304107.180

R Rana Rahman, N Verma, P Kumar, K Eastham. Department of Paediatrics, Sunderland Royal Hospital, Sunderland, UK

Introduction HHHFNC therapy is a modality of non-invasive ventilation which provides humidified, warm, high flow oxygen ( $>2$ litres per minute). It has been proposed as an alternative to CPAP in maintaining children without the need for intubation and mechanical ventilation. We document results of HHHFNC therapy on children with bronchiolitis in our big DGH.

Aim To review the effectiveness of HHHFNC therapy for patients with bronchiolitis.

Method A retrospective study analysing records of 27 paediatric patients treated with HHHFNC therapy who presented with bronchiolitis between September 2010 - March 2011. Diagnosis of bronchiolitis was made clinically, with the aid of nasopharyngeal aspirates. Measured variables were clinical state and capillary blood gas changes; pre and 12 hours on HHHFNC. CPAP was never used on these children. Data was of normal distribution hence paired t-test was used to determine statistical significance with SPSS software.

Results 27 patients (female- 14; male- 13; mean age- 2.4 months). Single or dual bronchiolitis viruses were detected in NPA samples in addition to a H1N1 strain in 3 patients.

Mean admission was 6 days with a mean duration on HHHFNC of 3.1 days and a median of 2 days. There was a mean reduction in respiratory rate count of 8.3; mean reduction in heart rate of 23.7 and $\mathrm{pH}$ values improved by a mean of 0.6 (CI 99\% 0.7). $65 \%$ patients changed from intravenous fluids or nasogastric feeds to oral by 24 hours.

The paired t-test analyses demonstrated statistical significance for changes in values for interval of difference of $\mathrm{pH}$ ( $\mathrm{p}$ value -0.016), respiration rate ( $p$ value- 0.001$)$ and heart rate ( $p$ value -0.00 ).

Conclusion Findings showed subjective and objective improvement in the patients' physical variables and blood gas analyses. There are huge potentials to cost savings and clinical effectiveness with $\mathrm{HHH}$ FNC therapy, as there was only one transfer to paediatric intensive care unit amongst these patients. Following the conclusions drawn from this data, a further prospective study aim would be to demonstrate whether HHHFNC has superiority or non-inferiority to CPAP therapy.

\section{British Association of Perinatal Medicine}

\section{G169 PREVENTING EARLY POSTNATAL HEAD GROWTH FAILURE IN VERY PRETERM INFANTS: THE RANDOMISED CONTROLLED SCAMP NUTRITION STUDY}

doi:10.1136/archdischild-2013-304107.181

${ }^{1} \mathrm{C}$ Morgan, ${ }^{1} \mathrm{P}$ McGowan, ${ }^{2} \mathrm{~S}$ Herwitker, ${ }^{3} \mathrm{AE}$ Hart, ${ }^{1,4} \mathrm{MA}$ Turner. ${ }^{1}$ Department of Neonatology, Liverpool Women's Hospital, Liverpool, UK; '2Department of Pharmacy, Royal Liverpool and Broadgreen University Hospitals, Liverpool, UK; ${ }^{3}$ School of Health and Medicine, University of Lancaster, Lancaster, UK; ${ }^{4}$ School of Women's and Child Health, University of Liverpool, Liverpool, UK

Background Postnatal head growth failure is well recognised in very preterm infants (VPI), the largest deficit occurs at 3-4 weeks followed by some catch-up growth until 36 weeks corrected gestational age (36wCGA). Head circumference (HC) is correlated with brain volume and later neurodevelopmental outcome. Early nutritional deficits commonly occur in parenteral nutrition (PN) dependent VPI. We hypothesised that a Standardised, Concentrated with Added Macronutrients Parenteral (SCAMP) nutrition regimen would improve early head growth.

Aim To compare the change in $\mathrm{HC}(\Delta \mathrm{HC})$ and standard deviation score $(\triangle \mathrm{SDS})$ achieved at day 28 in VPI randomised to receive SCAMP nutrition $(12 \%$ glucose, $3.8 \mathrm{~g} / \mathrm{kg} /$ day protein/lipid) or a control standardised, concentrated $\mathrm{PN}$ regimen $(10 \%$ glucose, $2.8 \mathrm{~g} / \mathrm{kg} /$ day protein/lipid)

Methods The double-blind study (ISRCTN: 76597892) received ethical approval. Control PN was started within 6 hours of birth. Following parental consent, VPI $(<1200$ g; $<29$ weeks) were randomised (day 2-5) to either start SCAMP or remain on the control regimen. HC was measured at randomisation, day 7 and then weekly until 36wCGA. Actual daily nutritional intake, biochemical and metabolic data were collected for day 1-28. Weekly growth data and major preterm complications were collected until 36wCGA.

Results 150/196 eligible infants were randomised at mean age 73.5 hours. Mean (SD) birthweight (g) and gestation (weeks) was: $900(158)$ versus 884(183) and 26.8(1.3) versus 26.6(1.4) in SCAMP $(\mathrm{n}=74)$ and control $(\mathrm{n}=76)$ groups respectively. SCAMP achieved higher mean actual protein/energy intakes (calculated weekly) with largest difference occurring in week $2: 25.5 \mathrm{~g} / \mathrm{kg}$ versus $20.9 \mathrm{~g} / \mathrm{kg}$ and; $762 \mathrm{kcal} / \mathrm{kg}$ versus $664 \mathrm{kcal} / \mathrm{kg}$. 